\title{
A Study of Clinical and Laboratory Profile of Dengue Fever in a Tertiary Care Teaching Hospital
}

\author{
Kamal Rajesh Jampana ${ }^{1}$, Atchuta Chytanya Paka ${ }^{2}$ \\ ${ }^{1}$ Associate Professor, Department of General Medicine, Nimra Institute of Medical Sciences, Nimra Nagar, Ibrahimpatnam, Jupudi, Vijayawada, Andhra \\ Pradesh-521456, ${ }^{2}$ Assistant Professor, Department of General Medicine, NRI Academy of Sciences, Mangalagiri Road, Chinakakani, Guntur, Andhra \\ Pradesh-522503.
}

\section{Abstract}

Background: The incidence of Dengue has grown dramatically around the world in recent decades. Year after year, the prevalence of the disease is on a rise. This study is designed to study the clinical and laboratory parameters of Dengue patients in a tertiary care teaching hospital. Subjects and Methods: Prospective observational study was undertaken in a tertiary care teaching hospital. This study was carried out for a period of two years between Jan 2017 to December 2018.Seventy-two patients were studied and analyzed. All patients with positive NS1 antigen and positive ELISA test were included in the study. Clinical, hematological and biochemical parameters were noted.Results: Of the 72 patients, majority were females $(52.8 \%)$. Fever was the most common symptom followed by other symptoms like head ache, myalgias etc. Conclusion: Dengue is an important cause of mortality and morbidity in our country. Early diagnosis and prompt management can have a positive effect on the outcome of the disease. Proper monitoring of the clinical and biochemical parameters and necessary intervention can bring down the mortality rates associated with this disease.

Keywords: NS1 antigen, Flavivirus, Thrombocytopenia.

Corresponding Author: Dr. Atchuta Chytanya Paka, Assistant Professor, Department of General Medicine, NRI Academy of Sciences, Mangalagiri Road, Chinakakani, Guntur, Andhra Pradesh-522503.

Received: October 2019

Accepted: October 2019

\section{Introduction}

Dengue is one of the most common arthropod-borne viral illnesses in humans. Dengue infects approximately 390 million people per year.Dengue has recently become a major public health problem in India causing significant morbidity, mortality and economic loss. There has been a recent resurgence of Dengue in India with wide variety of presentation ${ }^{[1]}$. This sudden emergence is due to unplanned urbanization and migration of population to urban areas. Dengue fever is caused by infection with one of the four serotypes ${ }^{[2]}$ of Dengue virus which is asingle stranded RNA virus of genus Flavivirus.

The WHO 2009 classification ${ }^{[3]}$ divides Dengue fever into two groups: uncomplicated and severe. The clinical presentation of Dengue fever is triphasic with febrile phase characterized by high fever, headache, myalgia, vomiting, joint pain, transient rash and mild bleeding manifestations. The Secondphase is marked by progression to severe Dengue characterized by plasma leakage induced shockand fluid accumulations (ascites or pleural effusion) with or without respiratory distress, severe bleeding and organ impairment.

Infection with one Dengue serotype confers lifelong homotypic immunity to that serotype and a brief periodof partial heterotypic immunity to other serotypes, but the patient can later be infected by all fourserotypes.

Dengue fevers are sometimes confused with Chikungunya infection. Although these two diseases share similar clinical features, prominent and prolonged joint pains are more consistent with Chikungunya whereas hemorrhagic manifestations are more common in Dengue. NS1 antigen detection may have higher sensitivity during the first five days after the onset of symptoms. Thereafter NS1 antigen levels decrease gradually and antibody detection tests have higher sensitivity after day 5 of infection.

Exact clinical and laboratory profile is crucial for diagnosis as well as successful management of the patients. In this study we evaluated patients with Dengue presenting to the emergency department of ourtertiary care hospital in a rural setting for clinical, hematological profile and management outcomes. This study is an attempt to elucidate the clinical and laboratory profile of serologically confirmed cases of Dengue fever in a tertiary care teaching hospital.

\section{Subjects and Methods}

This study was conducted in the Department of Medicine, Nimra Institute of Medical Sciences. This is an observational prospective study. Seventy-two patients with confirmed Dengue fever cases admitted to tertiary care teaching hospital over a period of two years during the Dengue fever 
season between Jan 2017 to December 2018 were included in this study. Patients presenting with symptoms like fever, rash, myalgia, dyspnea, retro orbital pain, seizures, pain abdomen and bleeding manifestations were admitted and further evaluated. NS1 Antigen, Dengue IgM and IgG Antibodies positive cases were included in the study. Commercially available one step immunochromatography assay was done and a positive ELISA Test was used for confirmation of cases. Patients who had scrub typhus, malaria and enteric fever were also included in this study. A complete blood count, liver function test, renal function test, chest x-ray, ultrasound abdomen and 2D echotests were also done. Patientsbelow the age of 15 were excluded from this study.

\section{Results}

A total of 72 cases admitted to our hospital between Jan 2017 to Dec 2018 were statistically analyzed. Most of the Dengue cases presented during the month of August and September which depicts the role of rainy season for case clustering during that season.

\begin{tabular}{|c|c|c|c|}
\hline \multicolumn{4}{|c|}{ Table 1: Age and Sex distribution of Dengue fever } \\
\hline \multirow{2}{*}{$\begin{array}{l}\text { Age Group (in } \\
\text { years) }\end{array}$} & \multicolumn{2}{|c|}{ Sex } & \multirow{2}{*}{$\begin{array}{c}\text { Total no. of } \\
\text { patients }\end{array}$} \\
\hline & Male & Female & \\
\hline $15-20 \mathrm{yrs}$ & 1 & 3 & $4(5.6 \%)$ \\
\hline $21-30 \mathrm{yrs}$ & 7 & 8 & $15(20.8 \%)$ \\
\hline $31-40 y r s$ & 8 & 10 & $18(25 \%)$ \\
\hline $41-50 \mathrm{yrs}$ & 6 & 8 & $14(19.4 \%)$ \\
\hline $51-60 y r s$ & 9 & 4 & $(18.1 \%)$ \\
\hline$>60 \mathrm{yrs}$ & 3 & 5 & $8(11.1 \%)$ \\
\hline Total no. of patients & $34(47.2 \%)$ & $38(52.8 \%)$ & 72 \\
\hline
\end{tabular}

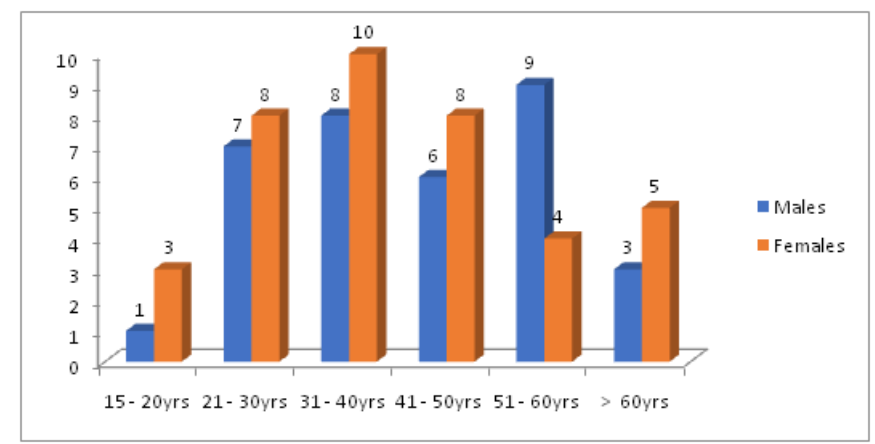

Chart 1: Age and Sex distribution of Dengue fever

Table 2: Presenting symptoms of Dengue fever

\begin{tabular}{|l|c|c|}
\hline \multicolumn{1}{|c|}{ Presenting symptom } & No. of patients & Percentage \\
\hline Fever & 70 & $97.2 \%$ \\
\hline Headache & 45 & $62.5 \%$ \\
\hline Myalgias & 30 & $41.7 \%$ \\
\hline Retro-orbital pain & 23 & $31.9 \%$ \\
\hline Pain abdomen & 20 & $27.8 \%$ \\
\hline Vomiting & 20 & $27.8 \%$ \\
\hline Shortness of breath & 11 & $15.3 \%$ \\
\hline Skin rash & 9 & $12.5 \%$ \\
\hline Jaundice & 2 & $2.8 \%$ \\
\hline Arthralgias & 1 & $1.4 \%$ \\
\hline Seizures & 1 & $1.4 \%$ \\
\hline
\end{tabular}

Table 3: Complications of Dengue fever
\begin{tabular}{|l|c|c|}
\hline \multicolumn{1}{|c|}{ Complication } & No. of patients & Percentage \\
\hline Capillary leak & 29 & $40.3 \%$ \\
\hline Dengue haemorrhagic fever & 15 & $22.2 \%$ \\
\hline Shock & 5 & $6.9 \%$ \\
\hline Encephalitis & 1 & $1.4 \%$ \\
\hline
\end{tabular}

\section{No. of patients}

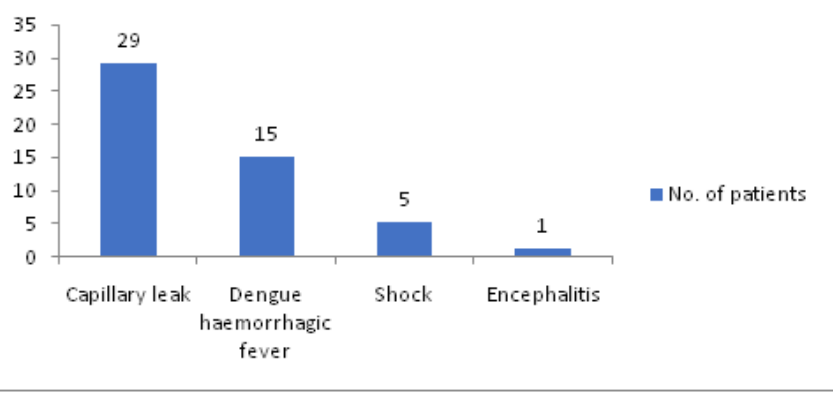

Chart 2: Complications of Dengue fever

Table 4: Ultrasound findings of Dengue fever

\begin{tabular}{|l|c|c|}
\hline \multicolumn{1}{|c|}{ Ultrasound finding } & No. of patients & Percentage \\
\hline Edematous gall bladder & 10 & $13.9 \%$ \\
\hline Hepatomegaly & 7 & $9.7 \%$ \\
\hline Ascites & 4 & $5.6 \%$ \\
\hline Pleural effusion & 2 & $2.8 \%$ \\
\hline Splenomegaly & 1 & $1.4 \%$ \\
\hline
\end{tabular}

Table 5: Laboratory findings of Dengue fever

\begin{tabular}{|l|c|c|}
\hline Laboratory finding & No. of patients & Percentage \\
\hline Thrombocytopenia & 71 & $98.6 \%$ \\
\hline Leucopenia & 40 & $55.6 \%$ \\
\hline$\uparrow$ SGOT levels & 17 & $23.6 \%$ \\
\hline$\uparrow$ SGPT levels & 12 & $16.7 \%$ \\
\hline$\uparrow$ Serum Creatinine levels & 6 & $8.3 \%$ \\
\hline$\uparrow$ Serum Bilirubin levels & 4 & $5.6 \%$ \\
\hline Abnormal PT \& INR levels & 3 & $4.2 \%$ \\
\hline
\end{tabular}

[Table-1 \& Chart-1] shows that $38(52.8 \%)$ of the cases were females and $34(47.2 \%)$ were males. $25 \%$ of the patients were of the age group of 31-40 years. All patients included in the study had fever which was the most common presenting symptom $(97.2 \%)$ followed by headache $(62.5 \%)$, myalgias (41.7\%),retro orbital pain(31.9\%), pain abdomen (27.8\%), vomiting $(27.8 \%)$, dyspnea (15.3\%), skin rash (12.5\%), jaundice (> 2.0 total bilirubin (2.8\%), joint pains $(1.4 \%)$ and seizures $(1.4 \%) .50$ of the patients had complications of which most common was capillary leak $(40.3 \%)$ followed by Dengue hemorrhagic fever (22.2\%), shock (6.9\%) and encephalitis (1.4\%).

Ultrasound findings noted in these patients included edematous gall bladder (13.9\%), hepatomegaly (9.7\%), ascites $(5.6 \%)$, pleural effusion $(2.8 \%)$ and splenomegaly $(1.4 \%)$. Out of the 72 patients, NS1 antigen test was found to be positive in 46 patients, $\operatorname{IgG}$ test was positive in 34 patients, IgM test was positive in 21 patients. Mixed infection (i.e. scrub typhus and malaria with Dengue positive) was noted in ten patients.

Laboratory parameter tests revealed leucopenia in 40 (55.6\%) patients and elevated SGOT in $17(23.6 \%)$ patients and elevated SGPT levels in 12 (16.7\%) patients. 
Thrombocytopenia was seen in 71 patients. Of the 71 patients with thrombocytopenia, 13 patients had platelet count $<1.5$ lakh, 19patients had platelet count between 50,000 to 1.0 lakh and 39 patients had platelet count less than 50,000. Bleeding manifestations were seen in 12 patients $(16.7 \%)$. Elevated serum Creatinine levels were noted in 6 patients $(8.3 \%)$. Serum bilirubin levels $>1.2$ levels were noted in 4 patients $(5.6 \%) .3$ patients $(4.2 \%)$ had abnormal PT and INR levels.

\section{Discussion}

Dengue is an important cause of mortality and morbidity in our country. It presents with a wide variety of clinicalmanifestations.Severity of the infectioncan range from influenza-like self-limiting illness to life-threatening complications like Dengue hemorrhagic fever (DHF) and Dengue shock syndrome.

Fever was the most common presentation (97.2\%), which is in unison with other similar studies from South East Asia $^{[4,5 \& 6]}$ Headache $(6162.5 \%)$ and myalgias $(41.7 \%)$ were the next two most common features/symptoms in the patients.

Liver dysfunction is common in Dengue and SGOT levels are usually higher than SGPT. Serum bilirubin levels> 1.2 levels were noted in 4 patients (5.6\%).Elevated serum Creatinine levels were noted in 6 patients (8.3\%). Ascites, pleural effusion, pericardial effusion and gallbladder wall edema are not uncommon in break bone fever. Hepatomegaly, splenomegaly, ascites and pleural effusion are usually mild and so are better detected by ultrasonography. In this study USG studies revealed ascites in $5.6 \%$ and pleural effusion in $2.8 \%$ of the patients.

Thrombocytopenia in Dengue can result in oxidative stress $^{[7]} .39$ of the patients in this study had platelet counts less than 50,000. Bleeding diathesis is a known feature of Dengue fever because of low platelet count and leakage from blood vessels.Bleeding manifestations were seen in $16.7 \%$ of the cases. Rashes are commonly associated with severe thrombocytopenia.

Rare but serious manifestations of Dengue include encephalitis myocarditis, ARDS, shock, liver failure. ${ }^{[8,9, ~ \& ~}$
${ }^{10]}$ Incidence of capillary leak, shock and encephalitis in the study was $40.3 \%, 6.9 \%$ and $1.4 \%$ respectively.

\section{Conclusion}

Maximum numbers of cases are seen in rainy season as water logging is the sourceof breeding ground for mosquitoes. Prevention of mosquito breeding in the monsoons can lead to fall in the incidence of Dengue cases in this season.In view of the increasing burden of Dengue on the public health-care system one should have a high index of suspicion. Early diagnosis and prompt intervention may help in reducing the mortality and morbidity.

\section{References}

1. World Health Organization. Dengue and Severe Dengue. Fact sheets, 2013.Available at: http://www.who.int/mediacentre/factsheets/fs117/ en /. Accessed 26 September 2013.

2. CDC. Imported Dengue--United States, 1997 and 1998. Morb Mortal Wkly Rep. 2000; 49:248-5

3. World Health Organization. Dengue: Guidelines for Diagnosis, Treatment, Prevention and Control, 2009. Available at: whqlibdoc.who.int/publications/2009/97892415478 71_eng.pdf.

4. Srikiathacorn A, Gibbons RV, Green S, Libraty DH, Thomos SJ, et al. Dengue hemorrhagic fever: The sensitivity and specificity of the World Health Organization definition for identification of severe cases of Dengue in Thailand, 1994-2005.Clin Infect Dis. 2010;50:1135-1143

5. Mohan D Kashinkunti, Shiddappa, Dhananjaya M. A Study of Clinical profile of Dengue fever in tertiary care teaching hospital Sch. J. App. Med. Sci., 2013;1:208-282.

6. Rachel Daniel, Rajamohanan and Aby Zachariah Philip. A Study of Clinical Profile of Dengue Fever in Kollam, Kerala, India. Dengue Bulletin. 2005; 29:197-202

7. Soundravally R, Sankar P, Bobby Z, Hoti SL. Oxidative stress in severe Dengue viral infection: association of thrombocytopenia with lipid peroxidation. Platelets. 2008; 19:447-54

8. Kumar R, Tripathi S, Tambe JJ, Arora V, Srivastava A, Nag VL. Dengue encephalopathy in children in Northern India: clinical features and comparison with non-Dengue. J Neurol Sci. 2008; 269:41-8

9. Kumar R, Tripathi S, Tambe JJ, Arora V, Nag VL. Dengue encephalopathy in children in Nothern India: Clinical features and comparison with non Dengue. J NeurolSci 2008; 269:41-8.

10. Jhamb R, Kashyap B, Ranga GS, Kumar A. Dengue fever presenting as acute liver failure - a case report. Asian Pac J Trop Med. 2011; 4:323-4

Copyright: (C) the author(s), 2019. It is an open-access article distributed under the terms of the Creative Commons Attribution License (CC BY 4.0), which permits authors to retain ownership of the copyright for their content, and allow anyone to download, reuse, reprint, modify, distribute and/or copy the content as long as the original authors and source are cited.

How to cite this article: Jampana KR, Paka AC. A Study of Clinical and Laboratory Profile of Dengue Fever in a Tertiary Care Teaching Hospital .Acad. J Med. 2019;2(2):18-20.

DOI: dx.doi.org/10.21276/ajm.2019.2.2.6

Source of Support: Nil, Conflict of Interest: None declared. 